Acta Crystallographica Section A

Foundations of

Crystallography

ISSN 0108-7673

\section{addenda and errata}

\title{
Fifty years of aperiodic crystals.
}

\section{Corrigendum}

\section{T. Janssen}

Institute for Theoretical Physics, University of Nijmegen, 6525 ED Nijmegen, The

Netherlands. Correspondence e-mail: t.janssen@science.ru.nl

Corrections to the article by Janssen [Acta Cryst. (2012). A68, 667-674] are given.

The following corrections should be made in the article by Janssen (2012):

(i) On page 671, right column, line 42 , and in the caption to Fig. 3, ' $n$-heptane-urea (Mariette et al., 2012)' should be changed to 'nonadecane-urea' (Toudic et al., 2011)'.

(ii) In the reference list, 'Mariette, C., Huard, M., Rabiller, P., Nichols, S. M. E., Colivet, C., Janssen, T., Alquist, K. E., Hollingsworth, M. D. \& Toudic, B. (2012). J. Chem. Phys. 136, 104505' should be changed to 'Toudic, B., Rabiller, P., Bourgeois, L., Huard, M., Ecolivet, C., McIntyre, G. J., Bourges, P., Breczewski, T. \& Janssen, T. (2011). Europhys. Lett. 93, 16003'.

\section{References}

Janssen, T. (2012). Acta Cryst. A68, 667-674.

Mariette, C., Huard, M., Rabiller, P., Nichols, S. M. E., Colivet, C., Janssen, T., Alquist, K. E., Hollingsworth, M. D. \& Toudic, B. (2012). J. Chem. Phys. 136, 104505.

Toudic, B., Rabiller, P., Bourgeois, L., Huard, M., Ecolivet, C., McIntyre, G. J., Bourges, P., Breczewski, T. \& Janssen, T. (2011). Europhys. Lett. 93, 16003. 